\title{
HUBE: THE HOPKINS ULTRAVIOLET BACKGROUND EXPERIMENT
}

\author{
Randy A. Kimble, ${ }^{1}$ Richard C. Henry, ${ }^{1}$ and Francesco Paresce ${ }^{1,2}$ \\ ${ }^{1}$ Center for Astrophysical Sciences \\ ${ }^{2}$ Space Telescope Science Institute \\ The Johns Hopkins University \\ Baltimore, MD 21218 USA \\ The Johns Hopkins University \\ Baltimore, MD 21218 USA
}

\begin{abstract}
The far-ultraviolet wavelength region between 1216 and $\sim 2000 \AA$ offers unusually favorable conditions for observing a variety of known or predicted diffuse emissions of both interstellar and extragalactic origin. We describe here a proposed instrument for studying those emissions, the Hopkins Ultraviolet Background Experiment (HUBE). HUBE consists of two compact, fast components: a broadband camera (1350-2000 $\AA$ ) with $2^{\prime}$ resolution and an imaging spectrograph (1230-1800 $\AA$ ) with $5 \AA$ resolution. These complementary components will be utilized for both a sky survey and for sensitive deep pointings. As either a free-flying Scout-class satellite or as an attached payload on the Space Station, HUBE will make possible an investigation of unprecedented scope into the sources of the far ultraviolet background.
\end{abstract}

\section{INTRODUCTION}

The 1216 to $2000 \AA$ wavelength range provides a remarkable window for studying diffuse emission processes in the universe. The advantages of this bandpass for cosmic background studies are twofold. On the one hand, the physical processes that are known or predicted to contribute significantly to the far-ultraviolet (UV) background are incredibly diverse; on the other hand, in no other UV or optical window are low surface brightness emissions from outside the solar system so readily observable.

The observability of the 1216 to $2000 \AA$ background arises from the relative absence of contamination due to airglow, zodiacal light, and stars. Longward of the bright $\mathrm{H}$ I Lyman $\alpha$ line at $1216 \AA$ (which can be rejected by crystalline windows), the only significant nighttime far-UV airglow emissions are the much fainter O I features at 1304 and $1356 \AA$. Zodiacal light, dropping in the far-UV along with the late-type solar spectrum, has never been detected shortward of $1800 \AA$. Lastly, the stellar radiation field in the far-UV arises from a small number of very luminous, galactic-plane-concentrated, early-type stars. Hence it is possible to observe diffuse emissions "between the stars," particularly at high galactic latitudes.

That there is something interesting to see in this observable window has been firmly established by a number of workers. For reviews and access to the literature, see Bowyer (1989, this volume) and Paresce (1989, this volume); for the most recent observational data, see Martin and Bowyer (1989b) and Murthy et al. (1989).

Within our own galaxy, contributions to the far-UV background are known to arise from a wide range of conditions in the interstellar medium. From the cold, neutral phase come dust-scattered starlight and $\mathrm{H}_{2}$ fluorescence (the latter recently detected near UV-bright stars by Witt et al. 1989 and in the diffuse interstellar medium by Martin, Hurwitz, and Bowyer 1989, this volume). From the hot, ionized phase comes thermal or resonantly scattered line emission (Jakobsen and Paresce 1981; Martin and Bowyer 1989b). 
However, while interstellar processes are known to be important in the far-UV background, they appear to be unable to account for the entire observed signal. Possible extragalactic sources for the residual include the integrated light of galaxies and quasars (the former constrained by Martin and Bowyer 1989a), as well as more exotic possibilities such as recombination radiation from a photo-ionized intergalactic medium or decay radiation from as yet unidentified nonbaryonic dark matter.

It is remarkable that the fundamentally different emission mechanisms outlined above are seen or predicted to be of roughly comparable intensity. The emission sources are nevertheless separable by virtue of their characteristic spectral signatures and spatial structures. A suitably designed all-sky survey mission would therefore make dramatic contributions to our understanding of the broad range of galactic and extragalactic sources indicated above.

We describe here a proposed mission to carry out such a survey: the Hopkins Ultraviolet Background Experiment. HUBE is intended to make a systematic investigation of the diffuse far-UV background in the wavelength range from 1230 to $2000 \AA$. To do so it will employ a pair of small telescopes, one feeding a long-slit imaging spectrograph, and one feeding a broadband camera. The spectrograph will perform $5 \AA$ resolution spectroscopy from 1230 to $1800 \AA$, while the camera will perform 2' imaging over a broad bandpass from 1350 to 2000 $\AA$. Together the two telescopes will perform a survey of the entire sky, along with numerous deep pointed observations to the telescopes' limits of sensitivity.

Both components of HUBE offer unique and powerful capabilities for the study of the UV background. The spectrograph will have the highest spectral resolution yet employed in a UV background experiment; high throughput coupled with long observing times will yield unprecedented sensitivity as well. The camera will derive the highest background sensitivity yet attained from its large field of view $\left(2.5^{\circ} \times 2.5^{\circ}\right)$ and long observing times, and it will be the first UV instrument with arcminute imaging to obtain significant sky coverage.

The complementary utilization of long-slit spectroscopy and broadband imaging is at the heart of the HUBE concept. The spectrograph provides the detailed astrophysical results of the experiment, distinguishing among the various sources and identifying what it is that the camera is mapping. In addition, the spectrograph will augment the camera performance by identifying and permitting correction for unexpected weak airglow emissions found within the camera bandpass.

The camera, on the other hand, will reveal the spatial structure of the various background sources. Its large grasp will provide the highest sensitivity for detecting the background in those directions where it is faintest and for mapping the background at the finest angular scale. In regions where the galactic contribution is faintest, the camera will probe the contributions of external galaxies through analysis of the spatial fluctuations in the background. Finally, the camera will augment the spectrograph performance by identifying and permitting the removal of even very faint stars which fall into the spectrograph entrance slit.

Within the currently planned NASA program, two very suitable opportunities for carrying out the HUBE mission exist: either as a Scout-class free-flyer in the Small Explorer program, or as an attached payload on the Space Station. Though the instrumental designs would differ in detail for the two options (the Space Station is able to accommodate larger payloads), our mission concept would remain the same. Because the flexibility (and timescale!) of a dedicated free-flyer would be optimal, we focus discussion on the Small Explorer option here. 


\section{INSTRUMENTATION}

The optical designs for the HUBE spectrograph and camera in the Scout-class option are shown in Figures 1 and 2, respectively.

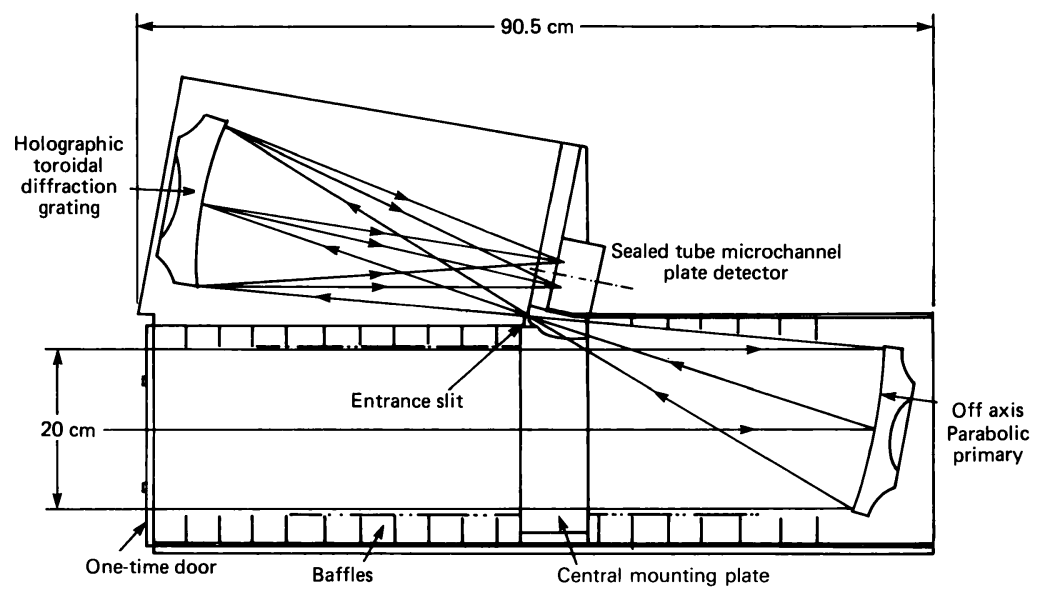

Figure 1. Optical layout of the HUBE long-slit spectrograph.

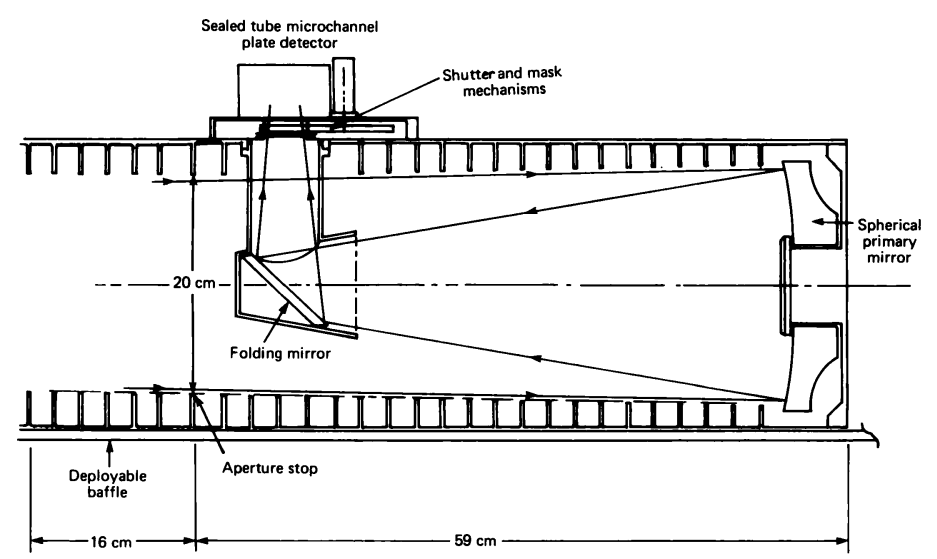

Figure 2. Optical layout of the HUBE broadband camera.

Each is simple, compact, and well within the state of the art for optics fabrication. The keys to their effectiveness as diffuse background instruments and as sky-survey instruments are speed, high throughput, and large format detectors (yielding sizable fields of view). We cannot detail the many design considerations here; we simply highlight some of the features that will enable HUBE to achieve unprecedented sensitivity: 
1. proper choice of bandpass (low unwanted backgrounds, high reflectivity optics);

2. arcminute imaging, enhancing detection and removal of point sources;

3. continuous monitoring of detector internal background using moving partial masks;

4. use of a holographically ruled diffraction grating for minimum scattering;

5. proper choice and location of crystal window bandpass filters to minimize particleinduced phosphorescence; and

6. proper choice of altitude $(500-550 \mathrm{~km})$ for avoidance of airglow and particle backgrounds.

The long-slit spectrograph is based on a $20 \mathrm{~cm}$ aperture, $\mathrm{f} / 2$ off-axis paraboloid, feeding the entrance slit $\left(2.1^{\prime} \times 2.5^{\circ}\right)$ of an $f / 2,40 \mathrm{~cm}$ diameter Rowland circle spectrograph. Within the spectrograph, a toroidal grating is employed, minimizing the astigmatism of a Rowland circle mount. Image quality along the slit ranges from $1^{\prime}$ to $10^{\prime}$ (including the aberrations of the primary); this will permit the signal from stars passing through the slit to be rejected with minimum loss of background signal.

Raytrace results indicate that the spectrograph will achieve slit-limited resolution (5 $\AA$ ) over the central $1.25^{\circ}$ of the field of view. Even at the edge of the slit, the resolution will be better ( $12 \AA$ at the worst) than has ever been applied to studies of the UV background.

The HUBE camera is a $64 \mathrm{~cm}$ focal length, $\mathrm{f} / 3.2$, Newtonian telescope with a $2.5^{\circ} \times 2.5^{\circ}$ field. The modest imaging requirements permit the use of a spherical primary, which can be superpolished to a low scattering surface, enhancing rejection of scattered light from bright stars outside of the field of view. We chose the Newtonian design for superior baffling of scattering paths than is possible for many fast designs.

Both the spectrograph and camera employ standard format, sealed tube, $40 \mathrm{~mm}$ diameter microchannel plate (MCP) detectors, which are identical except for the crystal windows mounted on the front $\left(\mathrm{CaF}_{2}\right.$ for the spectrograph and $\mathrm{BaF}_{2}$ for the camera). The photoncounting detectors have a solar-blind CsI photocathode deposited directly on the front MCP, and they utilize wedge-and-strip readouts (Martin et al. 1981). The resolution requirements for the detectors are well within the state of the art for these devices (e.g., Siegmund et al. 1986).

Uncertainties in the level of detector background have traditionally plagued diffuse background experiments. A key feature of the HUBE design is the use of partial masks for continuous internal background monitoring. At the spectrograph entrance slit, a rotating offcentered ring blocks a continuously shifting $20 \%$ portion of the entrance slit. At the camera focal plane, a bar mask is driven linearly back and forth across the field, again blocking $20 \%$ of the field at any given moment. Note that should a mechanism fail, there would simply be $20 \%$ of the field in constant shadow; the remaining $80 \%$ would be undisturbed.

\section{OBSERVING PROGRAM AND SENSITIVITY}

In the Small Explorer free-flyer option, the HUBE observing program will begin with deep pointings (typically $\geq 10,000 \mathrm{~s}$ ) of selected regions of known interest, e.g., toward the galactic poles, in directions of bright infrared cirrus emission, and over lines of sight of varying hydrogen column density. After two to three months of such pointings, a six-month spinning survey of the entire sky will begin. After the survey is complete, deep pointings will resume on additional interesting regions identified during the survey.

For the six-month sky-survey mode assumed, the camera's effective exposure time ranges from a minimum of $500 \mathrm{~s}$ for directions in the ecliptic plane to far longer at the ecliptic poles. The narrower field of view spectrograph has an effective integration time of $75 \mathrm{~s} \mathrm{deg}^{-2}$ in the 
ecliptic plane, increasing again toward the poles.

The resulting sensitivity for the HUBE broadband camera is summarized in Figure 3, which shows the minimum detectable flux at a signal-to-noise of 10 for both the sky survey mode and for $10,000 \mathrm{~s}$ deep pointings. We see that the minimum previously reported intensity of $\sim 300$ photons $\mathrm{cm}^{-2} \mathrm{~s}^{-1} \mathrm{sr}^{-1} \AA^{-1}$ could be detected at $10 \sigma$ in every $2^{\prime} \times 2^{\prime}$ resolution element over the entire sky, while far lower intensities are detectable with modest binning of the survey data or during the deep pointings.

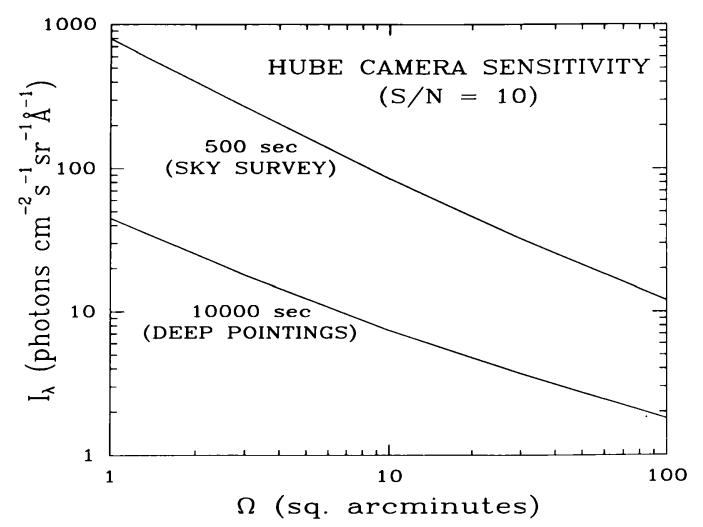

Figure 3. Minimum detectable intensity vs. solid angle for the HUBE camera.

The corresponding continuum sensitivity of the HUBE spectrograph is shown in Figure 4. As signal-to-noise can be improved equally well by increasing the observing time or by binning in wavelength, the minimum detectable intensity is plotted vs. $T_{o b s} \times \Delta \lambda / 5 \AA$. We see that the minimum previously observed intensity is detectable at $5 \AA$ resolution for 5,000 different $8 \mathrm{deg}^{2}$ resolution elements over the sky, while far lower intensities will be observable over the slit solid angle of $0.09 \mathrm{deg}^{2}$ during the deep pointings.

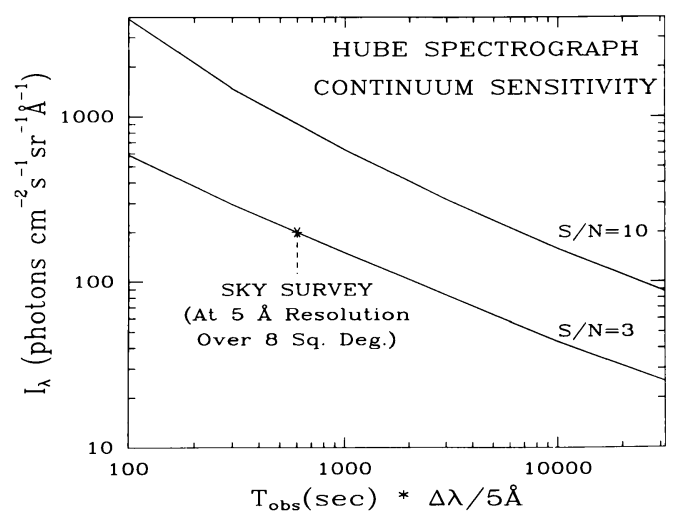

Figure 4. Minimum detectable intensity vs. observation time * wavelength binning for the HUBE spectrograph. 
Line detectability in the spectrograph is presented in Figure 5 as a function of observing time, for three different levels of continuum background, $I_{c}$, against which the lines must be viewed. Once again, the previously reported line intensities of thousands of photons $\mathrm{cm}^{-2} \mathrm{~s}^{-1} \mathrm{sr}^{-1}$ can be mapped with 5,000 resolution elements over the sky, while the deep pointings will bring out features down to a few hundred photons $\mathrm{cm}^{-2} \mathrm{~s}^{-1} \mathrm{sr}^{-1}$.

A more striking visual demonstration of HUBE capabilities is offered in Figure 6 by the simulated spectrum of a $10,000 \mathrm{~s}$ pointing. We show here the response of HUBE to a UV background composed of (i) 300 photons cm $\mathrm{cm}^{-2} \mathrm{~s}^{-1} \mathrm{sr}^{-1} \AA^{-1}$ of extragalactic and dust-scattered continuum radiation; (ii) high-ionization line emission predicted by the model of Paresce, Monsignori Fossi, and Landini (1983) as a "canonical galactic corona" and (iii) molecular

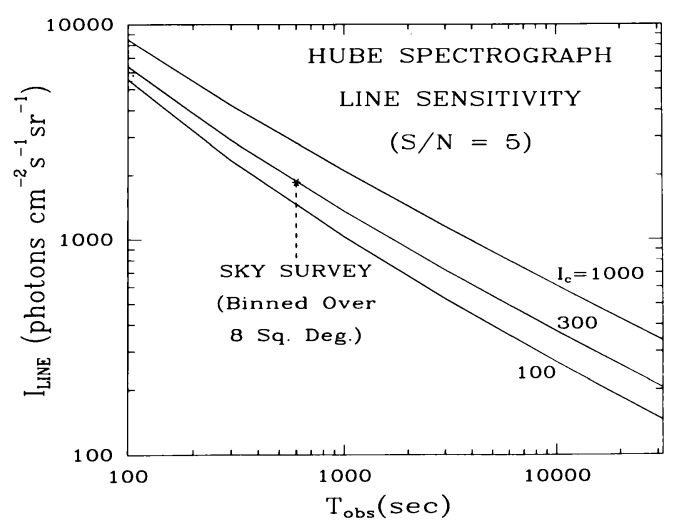

Figure 5. Minimum detectable line intensity vs. observation time for the HUBE spectrograph, plotted for three values of continuum background, $\mathrm{I}_{c}$.

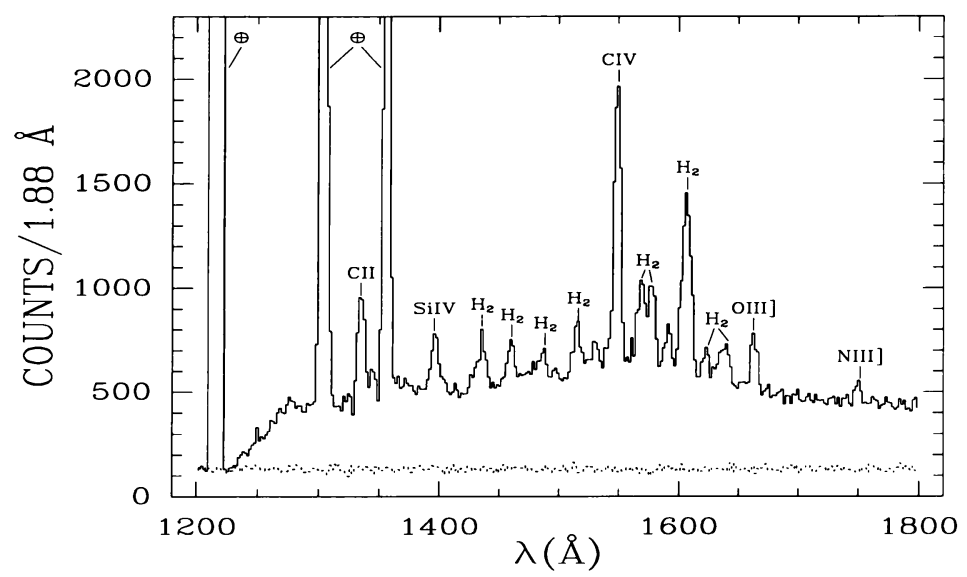

Figure 6. Simulated HUBE 10,000 s observation of the diffuse ultraviolet background. The dotted line indicates the contribution of the expected detector internal background to the observed signal; continuous monitoring of the internal background by means of a moving partial mask at the entrance slit will permit accurate subtraction of this component. 
hydrogen fluorescence at a level appropriate to high galactic latitudes according to the model of Jakobsen (1982). All three components are detected at high signal-to-noise, and there is no difficulty in separating the various contributors to the spectrum.

\section{SUMMARY}

We have described an instrument and a mission concept for carrying out an extensive, systematic investigation of the diffuse far ultraviolet background. This mission, the Hopkins Ultraviolet Background Experiment, is intended to go far beyond limited, short-duration studies done earlier, which have yielded nonetheless such tantalizing results. Even with previously achieved sensitivity and resolution, a full sky mapping of the already known contributors to the far-UV background would be of enormous value for a wide range of astrophysical studies done earlier. With the superior sensitivity and resolution of the HUBE sky survey, along with the far greater sensitivity of the HUBE deep pointings, who knows what may be found?

Acknowledgments-We thank our fellow members of the HUBE science team: Murk Bottema, Mark Clampin, Arthur Davidsen, Rodger Doxsey, Paul Feldman, Peter Jakobsen, Michael Jura, and Allan Sandage. We are also grateful to Glen Fountain and Thomas Strikwerda, who have led a major engineering definition effort for HUBE at the Johns Hopkins Applied Physics Laboratory.

\section{REFERENCES}

Bowyer, S. 1989, in Proc. IAU 139, Galactic and Extragalactic Background Radiation, ed. S. Bowyer and Ch. Leinert, Dordrecht, Kluwer Academic Publishers.

Jakobsen, P. 1982, Astr. Ap., 106, 375.

Jakobsen, P., and Paresce, F. 1981, Astr. Ap., 96, 23.

Martin, C., and Bowyer, S. 1989a, Ap. J., 338, 677.

Martin. C., and Bowyer, S. 1989b, Ap. J., in press.

Martin, C., Hurwitz, M., and Bowyer, S. 1989, in Proc. IAU 139, Galactic and Extragalactic Background Radiation, ed. S. Bowyer and Ch. Leinert, Dordrecht, Kluwer Academic Publishers.

Martin, C., Jelinsky, P., Lampton, M., Malina, R. F., and Anger, H. 1981, Rev. Sci. Instrum., 52, 1067.

Murthy, J., Henry, R. C., Feldman, P. D., and Tennyson, P. D. 1989, Ap. J., 336, 954.

Paresce, F. 1989, in Proc. IAU 139, Galactic and Extragalactic Background Radiation, ed. S. Bowyer and Ch. Leinert, Dordrecht, Kluwer Academic Publishers.

Paresce, F., Monsignori Fossi, B. C., and Landini, M. 1983, Ap. J. (Letters), 266, L107.

Siegmund, O. H. W., Lampton, M., Bixler, J., Chakrabarti, S., Vallerga, J., Bowyer, S., and Malina, R. F. 1986, J. Opt. Soc. Am. A, 3, 2139.

Witt, A. N., Stecher, T. P., Boroson, T. A., and Bohlin, R. C. 1989, Ap. J. (Letters), 336, L21.

J. M. Deharveng: You do not plan to observe the wavelength range between Lyo and the Lyman break. This range might be important for instance to see the decrease of the extragalactic background component if dominated by the integrated light of galaxies.

R. Kimble: The sub-Ly $\alpha$ UV background would indeed be of great interest, both for the reason you mention, and also for observing galactic emissions such as OVI. We have chosen a very conservative strategy for HUBE, preferring to concentrate on the region longward of Ly $\alpha$ and avoiding risks of Ly $\alpha$ scattering and the complications associated with windowless detectors. 